\title{
Correlated Polyhedral Rotations in the Absence of Polarons during Electrochemical Insertion of Lithium in $\mathrm{ReO}_{3}$
}

Nicholas H. Bashian, ${ }^{\dagger}$ Shiliang Zhou, ${ }^{\dagger}$ Mateusz Zuba, ${ }^{\ddagger}$ Alex M. Ganose, ${ }^{\natural,},, \|$ Joseph W. Stiles,

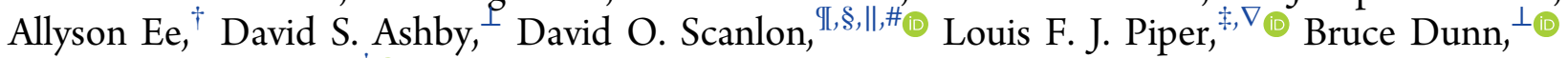
and Brent C. Melot*, ${ }^{\dagger}$

${ }^{\dagger}$ Department of Chemistry, University of Southern California, Los Angeles, California 90089, United States

${ }^{\ddagger}$ Department of Physics, Applied Physics and Astronomy, Binghamton University, Binghamton, New York 13902, United States

${ }^{\mathrm{II} D e p a r t m e n t}$ of Chemistry, University College London, 20 Gordon Street, London WC1H 0AJ, U.K.

${ }^{\S}$ Diamond Light Source Ltd., Diamond House, Harwell Science and Innovation Campus, Didcot, Oxfordshire OX11 0DE, U.K.

${ }^{\|}$Thomas Young Centre, University College London, Gower Street, London WC1E 6BT, U.K.

${ }^{\perp}$ Department of Materials Science and Engineering, University of California, Los Angeles, California 90095, United States

${ }^{\#}$ The Faraday Institution, Quad One, Harwell Science and Innovation Campus, Didcot OX11 0RA, U.K.

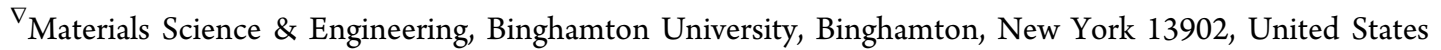

Supporting Information

ABSTRACT: Understanding the structural transformations that materials undergo during (de)insertion of $\mathrm{Li}$ ions is crucial for designing high-performance intercalation hosts as these deformations can lead to significant capacity fade. Herein, we present a study of the metallic defect perovskite $\mathrm{ReO}_{3}$ to determine whether these distortions are driven by polaronic charge transport (i.e., the electrons and ions moving through the lattice in a coupled way) due to the semiconducting nature of most oxide hosts. Employing numerous techniques, including electrochemical probes, operando $\mathrm{X}$-ray diffraction, $\mathrm{X}$-ray photoelectron spectroscopy, and density functional theory calculations, we find that the cubic structure of

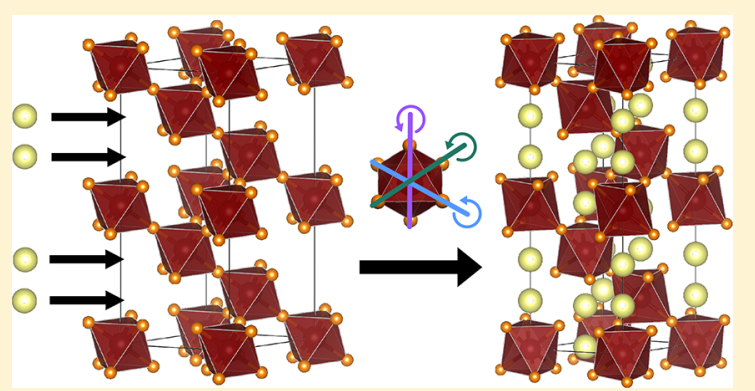
$\mathrm{ReO}_{3}$ experiences multiple phase changes involving the correlated twisting of rigid octahedral subunits upon lithiation. This results in exceptionally poor long-term cyclability due to large strains upon lithiation, even though metallic character is maintained throughout. This suggests that phase transformations during alkali ion intercalation are the result of local strains in the lattice and not exclusively due to polaron migration.

$\mathrm{T}$ he reversible (de)insertion of $\mathrm{Li}$ ions into densely packed intercalation hosts is a complex process of fundamental importance to rechargeable batteries. As positively charged ions move in and out of a structure, redoxactive transition-metal centers change their formal oxidation state and, in the process, adjust their bond lengths so as to maintain local charge neutrality. ${ }^{1,2}$ These complex structural distortions generate substantial strain in the lattice that manifests itself as large changes to the unit-cell volume during cycling, which can result in cracking of the electrode and delamination from the current collector, ultimately shortening the life of the battery. ${ }^{3,4}$ Developing a deeper understanding of these structural transformations and how they influence charge transport is therefore a critical open question for designing new intercalation hosts.
Our group recently demonstrated that the presence of rigid subunits within a structure necessitates the ability to undergo highly correlated polyhedral rotations in order to relieve the strain generated when ions are incorporated into the lattice. Contemporaneously, Banerjee and co-workers performed a detailed spectroscopic study on $\mathrm{Li}$ intercalation into $\mathrm{V}_{2} \mathrm{O}_{5}$ and identified a correlation between changes in the metal's first coordination shell and charge hopping through the lattice, suggesting the possibility that the rotations we had reported could be driven by polaronic charge transport. ${ }^{5}$ Intrigued, we initiated a study of the $A$-site vacant perovskite $\mathrm{ReO}_{3}$, one of

Received: July 9, 2018

Accepted: September 12, 2018

Published: September 12, 2018 
only a handful of metallic oxides that are known. The topology of the structure, illustrated in Figure 1, contains a perfectly

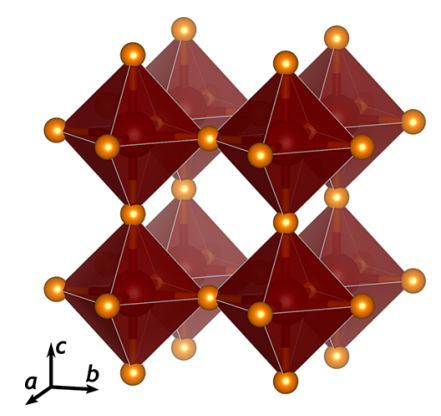

Figure 1. Cubic crystal structure of $\mathrm{ReO}_{3}$ with oxygen and rhenium ions shown in orange and red, respectively.

cubic network of corner-sharing octahedra of $\mathrm{ReO}_{6}$ with $90^{\circ}$ and $180^{\circ} \mathrm{O}-\mathrm{Re}-\mathrm{O}$ and $\mathrm{Re}-\mathrm{O}-\mathrm{Re}$ bond angles, respectively, creating a three-dimensional network of interstitials that Cava, Murphy, and co-workers reported can accommodate up to two $\mathrm{Li}$ ions per formula units (i.e., $\mathrm{Li}_{2} \mathrm{ReO}_{3}$ ) when treated with $n$ BuLi. ${ }^{6,7}$

The coexistence of intrinsic metallic conductivity and pathways for facile ionic diffusion make $\mathrm{ReO}_{3}$ a model system for exploring correlations between the transport of ions and electrons. While the open framework of $\mathrm{ReO}_{3}$ has been heavily investigated in the past for its negative thermal expansion properties, ${ }^{8,9}$ to our knowledge, there have been no studies that examine the role of structural flexibility on the performance of $\mathrm{ReO}_{3}$ as an intercalation electrode in functioning Li-ion batteries. Herein, we characterize the electrochemical performance using operando synchrotron Xray diffraction (XRD), density functional theory (DFT) calculations, and X-ray spectroscopy to better understand the fundamental mechanism for the (de)insertion of ions into the $\mathrm{ReO}_{3}$. Although the metallic character of the starting phase might be expected to minimize lattice deformations resulting from polaron migration, we observe pronounced rotations of the $\mathrm{Re}$ octahedra during (de)insertion of $\mathrm{Li}$. These tilting modes have a marked effect on the electronic structure of the material, eventually opening a small band gap in the fully lithiated $\mathrm{Li}_{2} \mathrm{ReO}_{3}$ end member. These distortions generate sufficient strain within the lattice to severely limit the reversible capacity over extended cycling, clearly demonstrating the importance of local structural distortions such as these for developing high-performance intercalation hosts. ${ }^{10}$

Several methods to prepare highly crystalline powders of $\mathrm{ReO}_{3}$ have been reported. ${ }^{11,12}$ The method of Chong et al. was chosen because of its ability to produce fairly monodispersed nanoparticles in a highly repeatable way, as illustrated in the inset of Figure 2. ${ }^{13}$ Immediate heating of the solution of $\mathrm{Re}_{2} \mathrm{O}_{7}$ produced nanoparticles with an average size around $15 \mathrm{~nm}$, and it was found that allowing the $\operatorname{Re}_{2} \mathrm{O}_{7}$ solution to sit on the bench prior to heating resulted in noticeably larger particles of $\mathrm{ReO}_{3}$. The observation of lattice fringes in the transmission electron microscopy images, shown as the inset of Figure 2, clearly demonstrates that even very brief heating times were sufficient to yield highly crystalline particles of $\mathrm{ReO}_{3}$. This is supported by the results of Rietveld refinement of the structure against synchrotron XRD patterns (Figure 2), which exhibit fairly sharp peaks with only very minor broadening of the reflections and no signs of any secondary phases. A

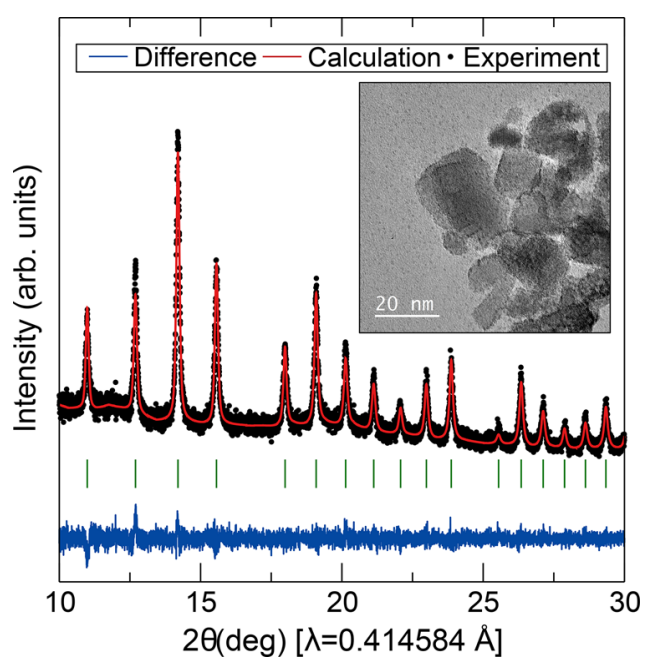

Figure 2. Results of the Rietveld refinement of the cubic structure against synchrotron XRD patterns of the nanoscale $\mathrm{ReO}_{3}$ samples where $R_{\text {Bragg }}=2.8 \%$. Detailed results of the refinement can be found in Table S2.

microstructural analysis of the peak broadening performed within FullProf indicated an average particle size of $20 \mathrm{~nm}$, in close agreement with the results of the microscopy. No significant differences were seen when cycling particles with larger sizes, so powders prepared in this way were used in all of the following discussion.

These powders were used to prepare film electrodes as described in the Experimental Details in the Supporting Information, and their galvanostatic performance was evaluated in standard Swagelok cells. During discharge, three distinct changes in slope can be seen at 2.5, 2.3, and $1.3 \mathrm{~V}$, yet only the first and last feature are distinguishable on reversal, as shown in Figure 3a. The presence of such a polarization implies a kinetic barrier to either electron or ion transport. The metallic character of $\mathrm{ReO}_{3}$ would seemingly preclude any issues with electronic transport, and therefore the polarization is likely related to sluggish Li-ion mobility or an asymmetric insertion process. Galvanostatic intermittent titration technique (GITT) experiments were therefore performed to determine how much of the polarization was due to nonequilibrium cycling conditions; however, a significant polarization is still seen even when the cell is allowed to fully relax (Figure S4).

The electrochemistry was further characterized using cyclic voltammetry in excess electrolyte in order to ensure (de)insertion was not limited by mass transport effects. Powders of $\mathrm{ReO}_{3}$ were deposited on strips of steel and characterized in a three-electrode configuration where an open circuit voltage near $3.4 \mathrm{~V}$ was observed. Figure $3 \mathrm{~b}$ shows the same three Faradaic peaks found in the galvanostatic cycling data between 1.0 and $4.0 \mathrm{~V}$. Yet, when sweeping across the entire voltage window, a partially irreversible wave evolves below $1.5 \mathrm{~V}$ that corresponds to the onset of electrolyte reduction to form $\mathrm{Li}_{2} \mathrm{CO}_{3}$ on the surface of the cathode, as will be shown more clearly later. ${ }^{14}$

In order to gain insight into the mechanism for Li-ion intercalation into the perovskite framework, operando X-ray diffraction experiments were performed at the 17-BM line at Argonne National Lab. The use of synchrotron X-rays allows for the collection of high-intensity diffraction patterns during 

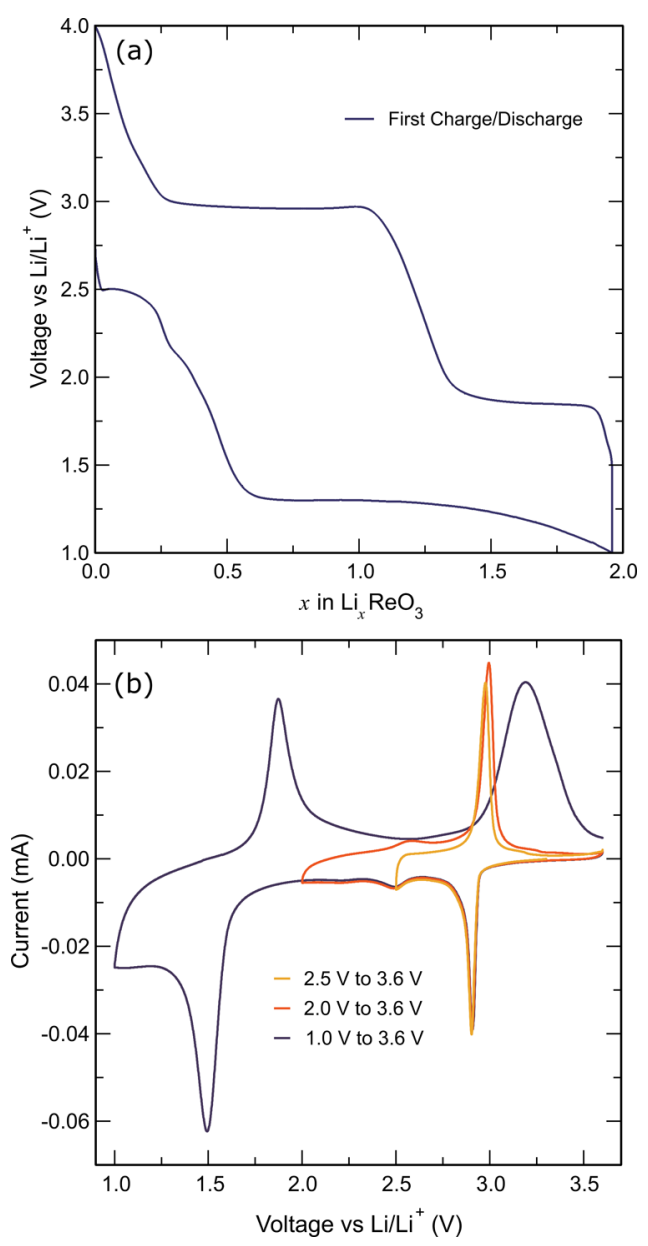

Figure 3. (a) Voltage vs composition of $\mathrm{Li}_{x} \mathrm{ReO}_{3}$ during first cycle. (b) Cyclic voltammetry scans of $\mathrm{ReO}_{3}$ microelectrodes collected at $1 \mathrm{mV} \mathrm{s}{ }^{-1}$ against $\mathrm{Li} / \mathrm{Li}^{+}$in a three-electrode cell within the glovebox.

cell operation, showing clear correlations between electrochemical behavior and structural changes. Operando methods are important for ensuring the structural changes can be accurately correlated with distinct electrochemical features. Details of operando XRD experiments are provided in the Experimental Details within the Supporting Information. Figure 4 displays diffraction patterns that were collected every $15 \mathrm{~min}$ during a full discharge and charge cycle of $\mathrm{Li}_{x} \mathrm{ReO}_{3}(0<x<2.0)$ at a $C / 10$ rate.

Starting on discharge, the Bragg reflections maintain a relatively constant intensity but shift to higher angles for $\mathrm{Li}_{x} \mathrm{ReO}_{3}(0<x<0.35)$, suggesting the cubic symmetry is maintained when small amounts of $\mathrm{Li}$ are inserted into the structure and that a solid solution can be obtained over this interval. Subsequently, the peaks associated with $\mathrm{ReO}_{3}$ begin to lose intensity and new reflections that can be indexed to the hexagonal structure of $\mathrm{Li}_{x} \mathrm{ReO}_{3}$ identifed by Cava et al. begin to grow. ${ }^{7}$ Counterintuitively, this structural distortion leads to a contraction of the unit cell volume by approximately 5\%, which is due to $\mathrm{Li}^{+}$ions being too small to fully fill the vacant $A$-site in the structure. Panels a and b of Figure 5 show that the transformation from the highly ordered cubic structure to the hexagonal phase is the result of octahedral tilting modes along the [111] direction of the cubic cell, while Figure S3 shows

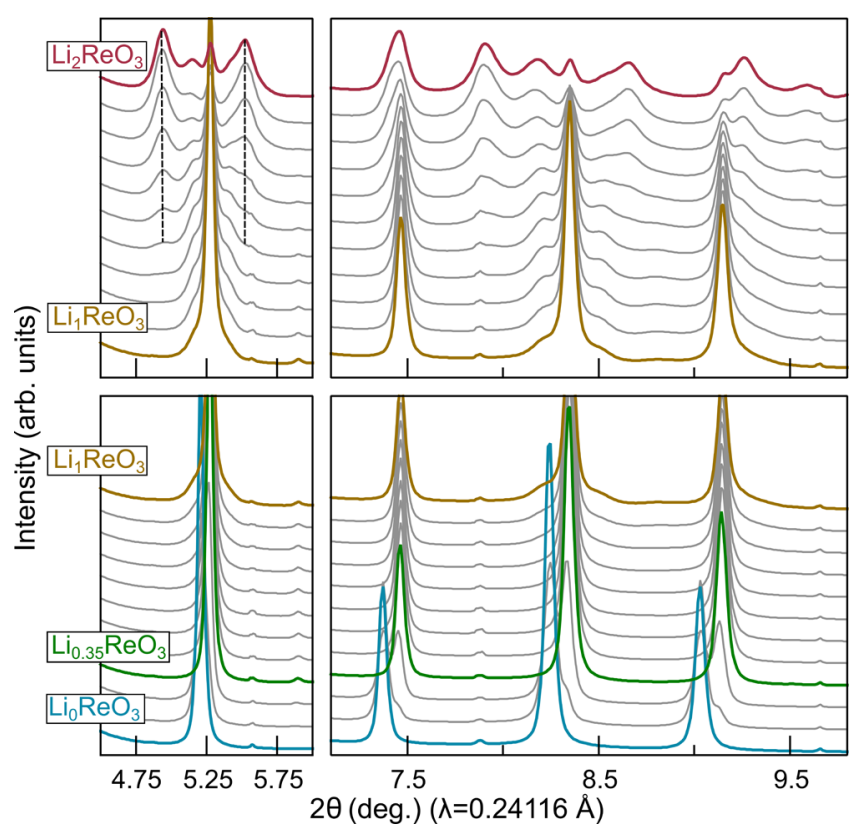

Figure 4. Operando synchrotron X-ray diffraction patterns of $\mathrm{ReO}_{3}$ throughout a complete discharge to $\mathrm{Li}_{2} \mathrm{ReO}_{3}$.

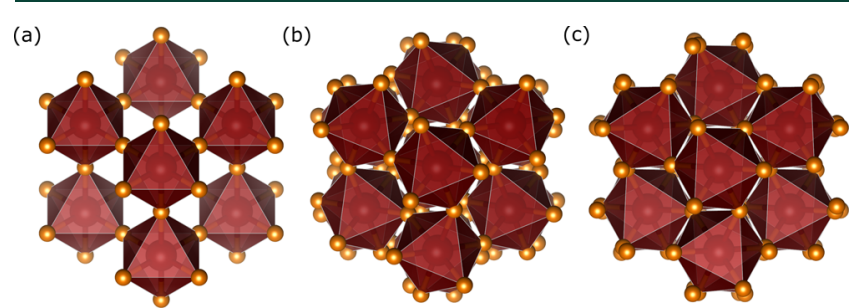

Figure 5. Illustration of the structural distortion that results in a twisting of the close-packed planes of oxygen in (a) $\mathrm{ReO}_{3}$, (b) $\mathrm{LiReO}_{3}$, and (c) $\mathrm{Li}_{2} \mathrm{ReO}_{3}$. Each structure is shown along the equivalent direction of the body diagonal within the cubic perovskite structure.

these rotations are accompanied by a contraction along the $c$ axis of the hexagonal cell.

As more $\mathrm{Li}$ is inserted, the peaks associated with the hexagonal phase remain essentially unchanged until approximately $x=1.25$, at which point new reflections begin to evolve until complete lithiation at $x=2.0$. This indicates that little structural rearrangement is required beyond the initial compression of the $A$-site pocket when only a single $\mathrm{Li}$ occupies each pocket. Yet, in order to insert beyond $x=1.0$, the $A$-site must re-expand to allow two $\mathrm{Li}$ ions per pocket, which is clearly seen as the peak at $5.25^{\circ}$ begins to evolve symmetric shoulders at $5.14^{\circ}$ and $5.41^{\circ}$ which continually increase their separation with increasing $\mathrm{Li}$ content. Interestingly, the $\mathrm{Li}_{x} \mathrm{ReO}_{3}$ phase never seems to entirely disappear, suggesting an incomplete discharge during the operando measurement. Unfortunately, because of the close structural relationship and extensive peak overlap in the patterns, precise Rietveld refinement of individual patterns proved intractable.

On charging, changes in the diffraction patterns mirror those in the discharge process albeit over a slightly different compositional range, as shown in Figure S5. This can be seen in the peaks at $5.2^{\circ}, 8.3^{\circ}$, and $9.1^{\circ}$ that are visible from 0.5 $>x>2.0$ compared to $2.0<x<1.25$ on discharge. The reason for the asymmetry in the structural distortions can be 
understood by examining Figures 5 and S3. Migration of $\mathrm{Li}$ into the cubic phase of $\mathrm{ReO}_{3}$ proceeds through large vacancies in a highly symmetric close-packed lattice, whereas the octahedral rotations that produce the $\mathrm{Li}_{2} \mathrm{ReO}_{3}$ structure close these vacancies and block many of the pathways for Li-ion transport. It should, therefore, not be surprising that such a complex rearrangement of atoms to return to cubic symmetry would proceed through a different mechanism that involves a more correlated shuffling of ions across each interstitial.

More importantly, these distortions severely limit the longterm cycling stability, as shown in Figure 6, where only $40 \%$ of

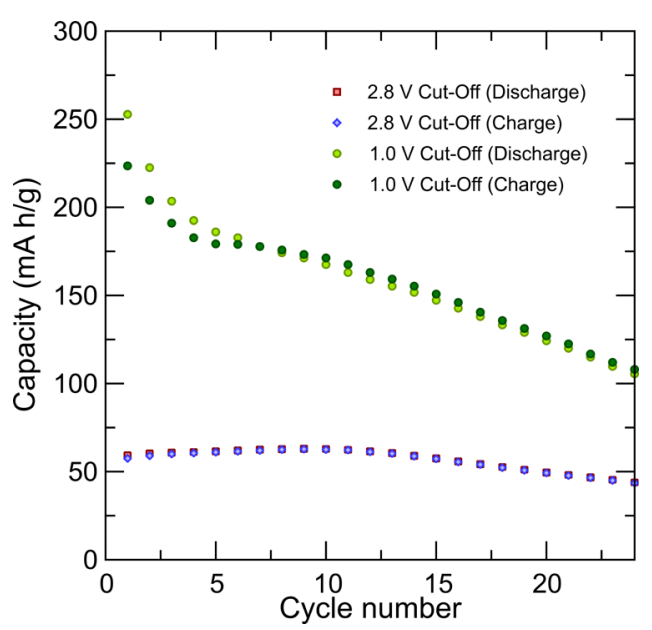

Figure 6. Charge and discharge capacity for a range of voltage cutoffs showing a severe loss of capacity when intercalating more than 0.35 equiv of $\mathrm{Li}$ ions into $\mathrm{ReO}_{3}$.

the initial capacity is retained after 25 cycles when the lower cutoff voltage is set at $1.0 \mathrm{~V}$. By setting the cutoff voltage to 2.8 $\mathrm{V}$, which avoids these distortions by only inserting lithium into the cubic form of $\mathrm{Li}_{x} \mathrm{ReO}_{3}(x<0.35)$, the capacity can be stabilized over multiple cycles. This may be expected as structural deformations are well-known to produce particle cracking and dewetting from the current collector that can electrically isolate portions of the electrode and reduce the accessible capacity of the cell. ${ }^{15-17}$ The severity of the effect is due to local strains as the corner-sharing octahedra rotate, bending the $\mathrm{O}-\mathrm{Re}-\mathrm{O}$ bond angle from $180^{\circ}$ to $145^{\circ}$ in $\mathrm{Li}_{x} \mathrm{ReO}_{3}$ and $138^{\circ}$ in $\mathrm{Li}_{2} \mathrm{ReO}_{3}$, corresponding a $23 \%$ change at full lithiation. The transformation to $\mathrm{Li}_{x} \mathrm{ReO}_{3}$ also results in a $5 \%$ contraction of the unit cell, yet further lithiation to $\mathrm{Li}_{2} \mathrm{ReO}_{3}$ acts to cancel this contraction by expanding to nearly the same volume as the Li-free starting material in order to accommodate the two $\mathrm{Li}$ ions per $A$-site. Interestingly, such pronounced structural transformations are somewhat unexpected given that nanoscale powders usually suppress phase transformations. ${ }^{18}$

Having firmly established that the structure of $\mathrm{ReO}_{3}$ distorts in a manner highly reminiscent to what has been reported for polyanionic intercalation hosts, the effect of these correlated rotations on the electronic structure was explored. As expected, hybrid density functional theory calculations indicate $\mathrm{ReO}_{3}$ is metallic, with three doubly degenerate bands $\left(\operatorname{Re} 5 \mathrm{~d}-\mathrm{t}_{2 \mathrm{~g}}\right.$ character), crossing the Fermi level, with the two Re $5 \mathrm{~d}-\mathrm{e}_{\mathrm{g}}$ bands found higher in energy $(\sim 4-6 \mathrm{eV}$ above the Fermi level), in agreement with previous calculations. ${ }^{19,20}$ In total, these six bands share a single electron, causing the Fermi level to sit resonant within them. The density of states (DOS) and band structure of for $\mathrm{ReO}_{3}, \mathrm{LiReO}_{3}$, and $\mathrm{Li}_{2} \mathrm{ReO}_{3}$ are shown in Figure $7 \mathrm{a}-\mathrm{f}$. The orbital projected DOS clearly shows the edge of the valence band maxima to be entirely composed of Re $5 \mathrm{~d}$ and $\mathrm{O} 2 \mathrm{p}$ orbitals, with the Re states dominating at the Fermi level.

Similar to $\mathrm{ReO}_{3}$, the valence band of $\mathrm{LiReO}_{3}$ is again composed of $\operatorname{Re} 5 \mathrm{~d}$ and $\mathrm{O} 2 \mathrm{p}$ orbitals, with the metallic behavior being conserved despite the significant buckling of the $\mathrm{Re}-\mathrm{O}$ bond angles. However, the rotations of the octahedra produce six significantly less disperse bands crossing the Fermi level in $\mathrm{ReO}_{3}$ to split into two groups: the first consisting of two bands, spanning the energy range -0.6 to 1.0 $\mathrm{eV}$, and the second consisting of four bands, spanning the energy range -2.0 to $-0.3 \mathrm{eV}$. Further distorting the lattice to $\mathrm{Li}_{2} \mathrm{ReO}_{3}$ results in a medium band gap semiconductor, with an indirect band gap of $2.38 \mathrm{eV}$ due to the full occupation of these six bands. These calculations indicate that $\mathrm{Li}_{x} \mathrm{ReO}_{3}$ remains metallic for most values of $x$ up until $x=2.0$, which completely fills the valence band, strongly supporting the fact that the rotational distortions that occur on $\mathrm{Li}$ insertion are independent of any kind of polaron migration.

In order to experimentally corroborate the calculated DOS and further demonstrate that polaronic effects are not present during $\mathrm{Li}$ insertion, X-ray photoemission spectroscopy was used to evaluate the electronic structure of the phases. Figure $8 \mathrm{a}$ shows the $\mathrm{Li} 1 \mathrm{~s}$ and $\mathrm{Re} 4 \mathrm{f}$ core regions of pristine and lithiated states of $\mathrm{ReO}_{3}$. Pristine $\mathrm{ReO}_{3}$ is expected to have a $\mathrm{Re}^{6+}$ oxidation state, with a $4 \mathrm{f}_{7 / 2}$ peak centered at $43.1 \mathrm{eV}$. The photoemission for $\mathrm{ReO}_{3}$ displays additional peaks associated with surface $\mathrm{Re}^{7+}$, because $\mathrm{Re}_{2} \mathrm{O}_{7}$ is the most thermodynamically stable phase and exists on the surface as $\operatorname{Re}^{7+}$ with its main peak at $45.5 \mathrm{eV}^{21}$ Upon lithiation, the Re $4 \mathrm{f}$ shifts to lower binding energy and reaches a state indicative of having a $\operatorname{Re}^{4+}$ oxidation state (main $4 \mathrm{f}_{7 / 2}$ peak at $42.2 \mathrm{eV}$ ). In addition, the main $\mathrm{Li} 1 \mathrm{~s}$ peak becomes present in the fully lithiated state. Because of the low discharge voltages, $\mathrm{Li}_{2} \mathrm{CO}_{3}$ and $\mathrm{LiF}$ contaminants contribute to this $\mathrm{Li} 1 \mathrm{~s}$ signal. These assignments were based on the $\mathrm{O} 1 \mathrm{~s}$ and F 1s core levels (see Figure S6). Given the fact that we can still see the $\operatorname{Re} 4 \mathrm{f}$, we can estimate the surface contaminants have a thickness of $\leq 3 \mathrm{~nm}$.

The valence band structure was measured for direct comparisons with the DFT calculations of the distorted $\mathrm{Li}_{x} \mathrm{ReO}_{3}$ phases, with their band structures and density of states plotted in Figure 7. Figure $8 \mathrm{~b}$ shows the valence band structure of $\mathrm{Li}_{x} \mathrm{ReO}_{3}$ samples. In order to directly compare our experimental results with DFT, we accounted for the orbital cross sections and experimental broadening. The projected orbital density of states were weighted by the corresponding photoionization cross section. ${ }^{22}$ Figure $8 \mathrm{c}$ shows the convoluted sum of orbital projections from DFT weighted by their corresponding photoionization cross-section convoluted with a Voigt profile $(0.1 \mathrm{eV}$ Lorentzian width, $0.5 \mathrm{eV}$ Gaussian width) to match experimental resolution. Excellent agreement between the measured and calculated electronic structure of $\mathrm{ReO}_{3}$ is observed; the $3-10 \mathrm{eV}$ range is associated with primarily $\mathrm{O} 2 \mathrm{p}$, and the topmost states up to the Fermi level are hybridized $\mathrm{Re} 5 \mathrm{~d}-\mathrm{O} 2 \mathrm{p}$. As $\mathrm{ReO}_{3}$ discharges, we see two additional features emerge at 4 and $9 \mathrm{eV}$ along with increased intensity up to the Fermi edge, consistent with the DFT of $\mathrm{LiReO}_{3}$. Upon discharge to $2 \mathrm{Li}^{+}$, we note differences between the XPS and DFT due to the aforementioned surface species. Figure $8 \mathrm{~b}$ includes reference spectra of $\mathrm{Li}_{2} \mathrm{CO}_{3}$ and 

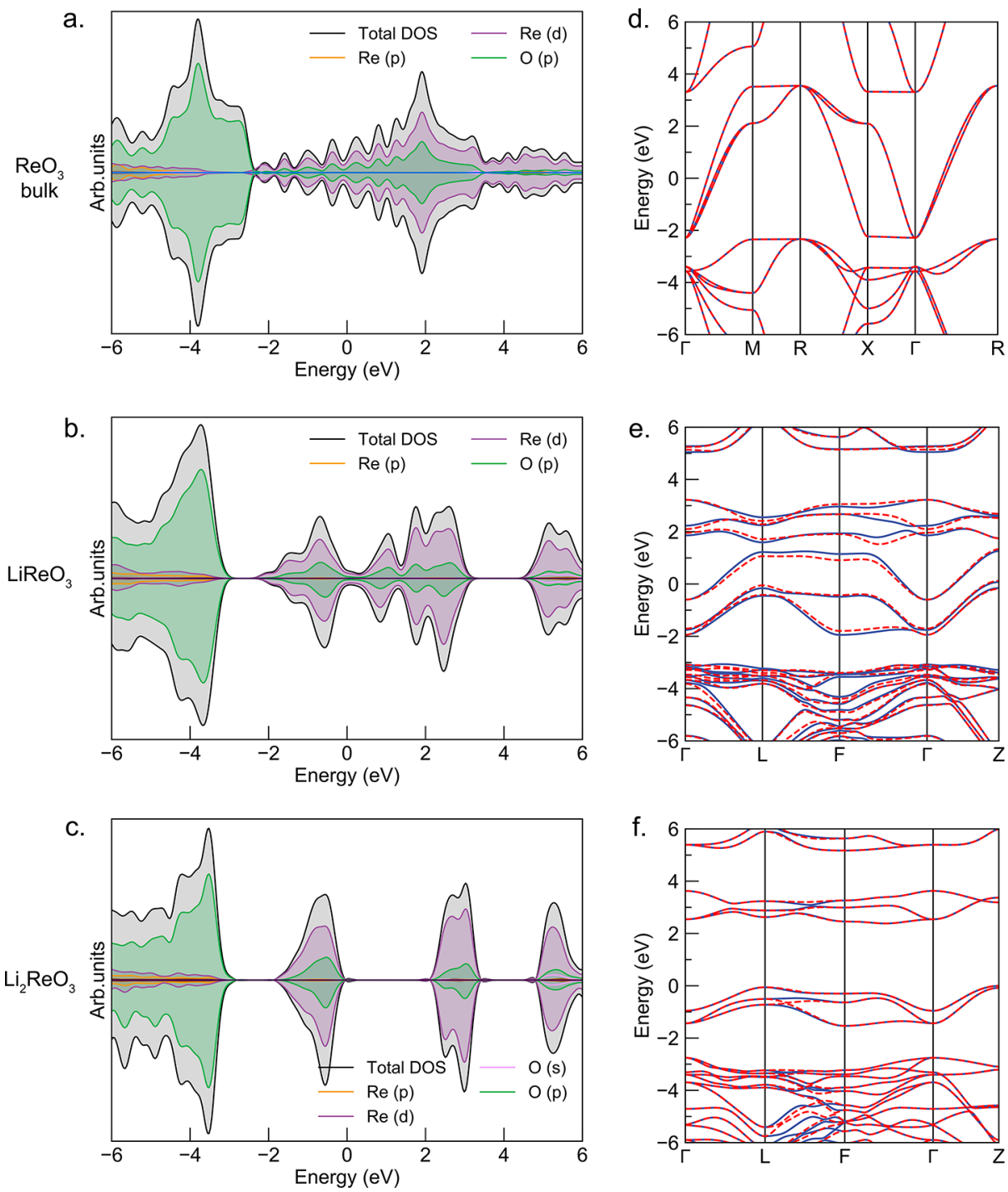

Figure 7. $(a-c)$ Densities of states and $(d-f)$ band structure for $\mathrm{ReO}_{3}, \mathrm{LiReO}_{3}$, and $\mathrm{Li}_{2} \mathrm{ReO}_{3}$.
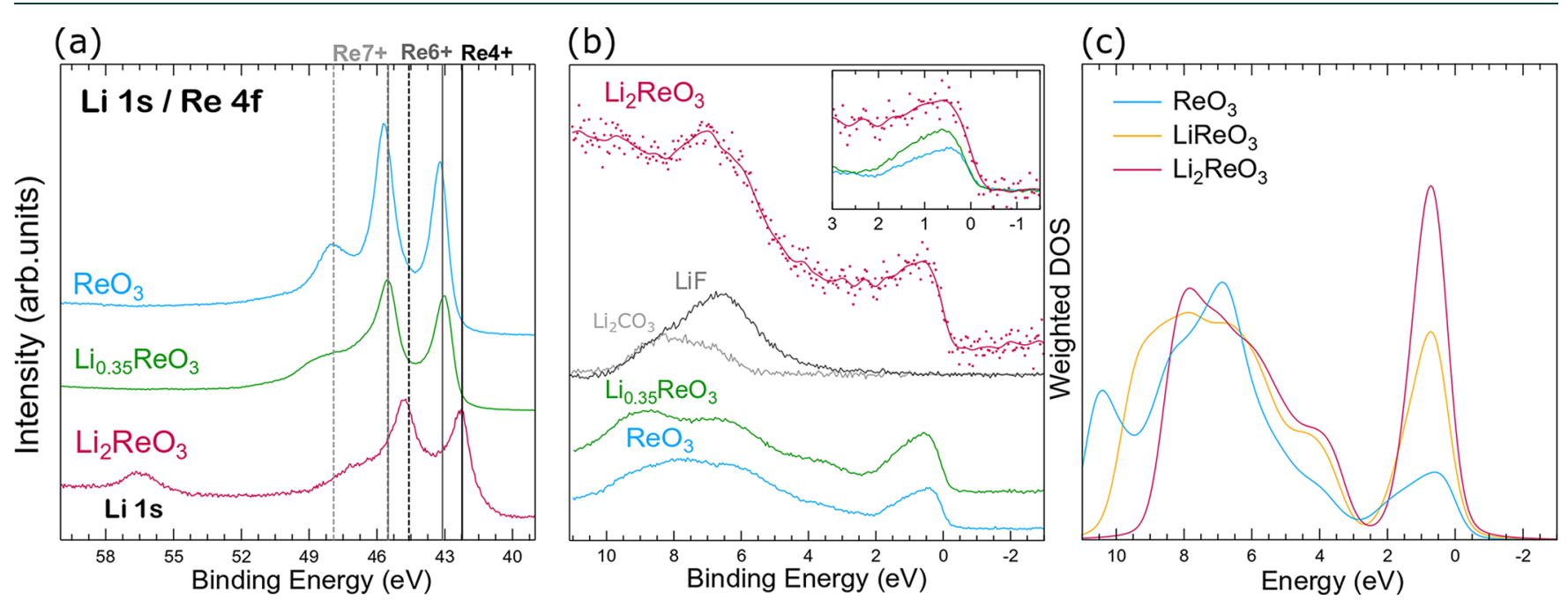

Figure 8. XPS of pristine $\mathrm{ReO}_{3}$ and lithiated states $\left(\mathrm{Li}_{0.35} \mathrm{ReO}_{3}\right.$ and $\left.\mathrm{Li}_{2} \mathrm{ReO}_{3}\right)$. (a) $\mathrm{Re} 4 \mathrm{f}$ core region with vertical line depicting $\mathrm{Re}$ oxidation state. (b) Valence band of $\mathrm{O} 2 \mathrm{p}$ and Re 5d hybridized regions with inset of Fermi level. (c) Weight and broadened density of states from DFT of $\mathrm{ReO}_{3}, \mathrm{LiReO}_{3}$, and $\mathrm{Li}_{2} \mathrm{ReO}_{3}$.

$\mathrm{LiF}$, which are seen to increase the spectral weight in the $6-10$ $\mathrm{eV}$ range. These surface species attenuate the $\mathrm{Li}_{2} \mathrm{ReO}_{3}$ signal and partially account for the poorer statistics for $\mathrm{Li}_{2} \mathrm{ReO}_{3}$ despite the longer data acquisition time taken for this sample. 
Despite these attenuating surface species, one still observes a very pronounced hybridized $\mathrm{Re} 5 \mathrm{~d}-\mathrm{O} 2 \mathrm{p}$ contribution in the XPS for $\mathrm{Li}_{2} \mathrm{ReO}_{3}$ (see the inset of Figure $8 \mathrm{~b}$ ), consistent with a fully occupied band. The poor photoemission signal of $\mathrm{Li}_{2} \mathrm{ReO}_{3}$ is consistent with the medium gap semiconducting character determined from the DFT calculations.

When all these results are combined, the metallic character of $\mathrm{ReO}_{3}$ appears to be exceptionally robust toward bending of the $\mathrm{O}-\mathrm{Re}-\mathrm{O}$ bond angle. While in the metallic state, electron transport is fully decoupled from the motion of the intercalating ions, which appears to indicate that polaron migration is not driving the correlated rotations of the cornersharing octahedra.

Using complementary electrochemical, spectroscopic, and structural characterization tools we have presented a detailed study on the mechanism of Li-ion insertion into $\mathrm{ReO}_{3}$. While the open framework and metallic character of $\mathrm{ReO}_{3}$ should, intuitively, allow it to exhibit exceptionally high performance as a cathode for Li-ion batteries, our results clearly show poor long-term cycling stability related to the highly correlated rotations of the corner-sharing perovskite octahedra. This work highlights the importance of understanding the underlying structural distortions that occur upon guest ion insertion during electrochemical cycling and, furthermore, demonstrates that the rotational distortions observed in insulating polyanionic intercalation hosts do not appear to be purely caused by polaron migration. These structural distortions severely impede the reversible (de)insertion of $\mathrm{Li}$ into $\mathrm{ReO}_{3}$ and would have to be suppressed in order for similar $A$-site vacant perovskite hosts to be effective electrode materials.

\section{ASSOCIATED CONTENT}

\section{S Supporting Information}

The Supporting Information is available free of charge on the ACS Publications website at DOI: 10.1021/acsenergylett.8b01179.

Experimental details, Rietveld refinement results, illustration of structural distortions, GITT measurements, operando XRD, and XPS measurements (PDF)

\section{AUTHOR INFORMATION}

\section{Corresponding Author}

*E-mail: melot@usc.edu.

\section{ORCID}

Shiliang Zhou: 0000-0003-3851-5176

David O. Scanlon: 0000-0001-9174-8601

Louis F. J. Piper: 0000-0002-3421-3210

Bruce Dunn: 0000-0001-5669-4740

Brent C. Melot: 0000-0002-7078-8206

\section{Notes}

The authors declare no competing financial interest.

\section{ACKNOWLEDGMENTS}

B.C.M. acknowledges support through a CAREER award from the National Science Foundation under Grant No. DMR1554204 for the electrochemical studies and Research Corporation for Science Advancement for a Cottrell Scholar award. B.C.M. and L.F.J.P also thank Research Corporation for Science Advancement for funding through the Scialog program. M.Z. was supported as part of the Multidisciplinary GAANN in Smart Energy Materials, a Graduate Areas of
National Need, funded by the U.S. Department of Education, under Award No. P200A150135 This work made use of the ARCHER UK National Supercomputing Service (http://www. archer.ac.uk), via membership of the UK's HEC Materials Chemistry Consortium, which is funded by EPSRC (EP/ L000202). D.O.S. acknowledges support from the EPSRC (EP/N001982/1 and EP/P00315X/1) and membership in the Materials Design Network. A.M.G. thanks Diamond Light Source for the cosponsorship of a studentship on the EPSRC Centre for Doctoral Training in Molecular Modeling and Materials Science (EP/L015862/1). Additional support was received from the Faraday Institution (Grant No. FIRG003). D.S.A. and B.D. were supported by the Nanostructures for Electrical Energy Storage (NEES), an Energy Frontier Research Center (EFRC) funded by the U.S. Department of Energy, Office of Science, Office of Basic Energy Sciences under Award Number DESC0001160. Use of the Advanced Photon Source at Argonne National Laboratory was supported by the U.S. Department of Energy, Office of Science, Office of Basic Energy Sciences, under Contract No. DE-AC0206CH11357.

\section{REFERENCES}

(1) Zhou, S.; Barim, G.; Morgan, B. J.; Melot, B. C.; Brutchey, R. L. Influence of Rotational Distortions on $\mathrm{Li}^{+}$- and $\mathrm{Na}^{+}$-Intercalation in Anti-NASICON Fe $2\left(\mathrm{MoO}_{4}\right)_{3}$. Chem. Mater. 2016, 28, 4492-4500.

(2) Barim, G.; Cottingham, P.; Zhou, S.; Melot, B. C.; Brutchey, R. $\mathrm{L}$. Investigating the Mechanism of Reversible Lithium Insertion into Anti-NASICON $\mathrm{Fe}_{2}\left(\mathrm{WO}_{4}\right)_{3}$. ACS Appl. Mater. Interfaces 2017, 9, 10813-10819.

(3) Bareño, J.; Shkrob, I. A.; Gilbert, J. A.; Klett, M.; Abraham, D. P. Capacity Fade and Its Mitigation in Li-Ion Cells with Silicon-Graphite Electrodes. J. Phys. Chem. C 2017, 121, 20640-20649.

(4) Feng, Y.; Dou, S.; Wei, Y.; Zhang, Y.; Song, X.; Li, X.; Battaglia, V. S. Preparation and Capacity-Fading Investigation of PolymerDerived Silicon Carbonitride Anode for Lithium-Ion Battery. ACS Omega 2017, 2, 8075-8085.

(5) De Jesus, L. R.; Horrocks, G. A.; Liang, Y.; Parija, A.; Jaye, C.; Wangoh, L.; Wang, J.; Fischer, D. A.; Piper, L. F. J.; Prendergast, D.; Banerjee, S. Mapping Polaronic States and Lithiation Gradients in Individual $\mathrm{V}_{2} \mathrm{O}_{5}$ Nanowires. Nat. Commun. 2016, 7, 12022.

(6) Cava, R.; Santoro, A.; Murphy, D.; Zahurak, S.; Roth, R. The Structures of Lithium-Inserted Metal Oxides: $\mathrm{LiReO}_{3}$ and $\mathrm{Li}_{2} \mathrm{ReO}_{3}$. J. Solid State Chem. 1982, 42, 251-262.

(7) Cava, R.; Santoro, A.; Murphy, D.; Zahurak, S.; Roth, R. The Structures of the Lithium Inserted Metal Oxides $\mathrm{Li}_{0.2} \mathrm{ReO}_{3}$ and $\mathrm{Li}_{0.36} \mathrm{WO}_{3}$. J. Solid State Chem. 1983, 50, 121-128.

(8) Rodriguez, E. E.; Llobet, A.; Proffen, T.; Melot, B. C.; Seshadri, R.; Littlewood, P. B.; Cheetham, A. K. The Role of Static Disorder in Negative Thermal Expansion in $\mathrm{ReO}_{3}$. J. Appl. Phys. 2009, 105, 114901.

(9) Chatterji, T.; Henry, P. F.; Mittal, R.; Chaplot, S. L. Negative Thermal Expansion of $\mathrm{ReO}_{3}$ : Neutron Diffraction Experiments and Dynamical Lattice Calculations. Phys. Rev. B: Condens. Matter Mater. Phys. 2008, 78, 134105.

(10) Melot, B. C.; Scanlon, D. O.; Reynaud, M.; Rousse, G.; Chotard, J.-N.; Henry, M.; Tarascon, J.-M. Chemical and Structural Indicators for Large Redox Potentials in Fe-Based Positive Electrode Materials. ACS Appl. Mater. Interfaces 2014, 6, 10832-10839.

(11) Biswas, K.; Rao, C. N. R. Metallic $\mathrm{ReO}_{3}$ Nanoparticles. J. Phys. Chem. B 2006, 110, 842-845.

(12) Nechamkin, H.; Kurtz, A. N.; Hiskey, C. F. A Method for the Preparation of Rhenium (VI) Oxide. J. Am. Chem. Soc. 1951, 73, 2828-2831.

(13) Chong, Y. Y.; Fan, W. Y. Facile Synthesis of Single Crystalline Rhenium (VI) Trioxide Nanocubes with High Catalytic Efficiency for 
Photodegradation of Methyl Orange. J. Colloid Interface Sci. 2013, 397, 18-23.

(14) Quackenbush, N. F.; Wangoh, L.; Scanlon, D. O.; Zhang, R.; Chung, Y.; Chen, Z.; Wen, B.; Lin, Y.; Woicik, J. C.; Chernova, N. A.; Ong, S. P.; Whittingham, M.; Piper, L. F. J. Interfacial Effects in $\epsilon$ $\mathrm{Li}_{x} \mathrm{VOPO}_{4}$ and Evolution of the Electronic Structure. Chem. Mater. 2015, 27, 8211-8219.

(15) Wang, H.; Jang, Y.; Huang, B.; Sadoway, D. R.; Chiang, Y. TEM Study of Electrochemical Cycling Induced Damage and Disorder in $\mathrm{LiCoO}_{2}$ Cathodes for Rechargeable Lithium Batteries. J. Electrochem. Soc. 1999, 146, 473-480.

(16) Dokko, K.; Nishizawa, M.; Horikoshi, S.; Itoh, T.; Mohamedi, M.; Uchida, I. In Situ Observation of $\mathrm{LiNiO}_{2}$ Single-Particle Fracture during Li-Ion Extraction and Insertion. Electrochem. Solid-State Lett. 2000, 3, 125-127.

(17) Jang, Y.-I.; Huang, B.; Wang, H.; Sadoway, D. R.; Ceder, G.; Chiang, Y.-M.; Liu, H.; Tamura, H. $\operatorname{LiAl}_{y} \mathrm{Co}_{1-y} \mathrm{O}_{2}(\mathrm{R} \overline{3} \mathrm{~m})$ Intercalation Cathode for Rechargeable Lithium Batteries. J. Electrochem. Soc. 1999, 146, 862-868.

(18) Kim, H.-S.; Cook, J. B.; Tolbert, S. H.; Dunn, B. The Development of Pseudocapacitive Properties in Nanosized- $\mathrm{MoO}_{2}$. J. Electrochem. Soc. 2015, 162, A5083-A5090.

(19) Mattheiss, L. Band Structure and Fermi Surface of $\mathrm{ReO}_{3}$. Phys. Rev. 1969, 181, 987.

(20) Cora, F.; Stachiotti, M.; Catlow, C.; Rodriguez, C. Transition metal oxide chemistry: Electronic structure study of $\mathrm{WO}_{3}, \mathrm{ReO}_{3}$, and $\mathrm{NaWO}_{3}$. J. Phys. Chem. B 1997, 101, 3945-3952.

(21) Greiner, M. T.; Rocha, T. C.; Johnson, B.; Klyushin, A.; KnopGericke, A.; Schlögl, R. The Oxidation of Rhenium and Identification of Rhenium Oxides During Catalytic Partial Oxidation of Ethylene: An In-Situ XPS Study. Z. Phys. Chem. 2014, 228, 521-541.

(22) Scofield, J. H. T. Theoretical Photoionization Cross Sections from 1 to $1500 \mathrm{keV}$; UCRL-51326; Lawrence Livermore Laboratory, 1973. 